\title{
Teledermatology Addressing Disparities in Health Care Access: a Review
}

\author{
Spandana Maddukuri ${ }^{1,2} \cdot$ Jay Patel ${ }^{1,2} \cdot$ Jules B. Lipoff $^{2}$ (D) \\ Accepted: 24 February 2021 / Published online: 12 March 2021 \\ (C) The Author(s), under exclusive licence to Springer Science+Business Media, LLC, part of Springer Nature 2021
}

\begin{abstract}
Purpose of Review Dermatologists have been at the forefront of researching telemedicine to expand access to care. The current COVID-19 pandemic has prompted even greater expansion and implementation of teledermatology. This review discusses the research examining the potential impact of teledermatology addressing disparities in care.

Recent Findings Teledermatology appears to increase access to dermatology given expanded means to deliver care. Specifically, recent studies have found increased access among Medicaid-insured, resource-poor urban and rural, and elderly populations. Teledermatology implementation also facilitates education among providers at different levels of training. Still, as some patients have inconsistent access to the required technology, increased reliance on telemedicine may also potentially increase disparities for some populations.

Summary Teledermatology may serve to reduce disparities in health care access in many underserved and marginalized communities. Future research should continue to study implementation, especially given the expansion during the COVID-19 pandemic. Ultimately, teledermatology may play an important role in ensuring equitable care access for all.
\end{abstract}

Keywords Teledermatology $\cdot$ Disparities $\cdot$ Health equity $\cdot$ Access to care $\cdot$ Telemedicine $\cdot$ Digital divide

\section{Introduction}

Telemedicine has grown in use since its inception in the early 1970s, and especially during the COVID-19 pandemic because of its ability to bridge barriers and gaps in care. Using visual communication, patient information, and interactive audio to conduct remote consultation, diagnosis, and treatment $[1,2]$, telemedicine is well-suited for the visually demanding specialty of dermatology $[3,4]$. As a subset of telemedicine, teledermatology is an effective and reliable mechanism to provide high-quality as well as cost- and timeeffective care for remote populations [5, 6]. It follows that teledermatology has been increasingly used to mitigate disparities in access to dermatologic care, which remains a major

This article is part of the Topical Collection on Teledermatology

Jules B. Lipoff

jules.lipoff@pennmedicine.upenn.edu

1 Corporal Michael J. Crescenz Veterans Affairs Medical Center, Philadelphia, PA, USA

2 Department of Dermatology, Perelman School of Medicine, University of Pennsylvania, Philadelphia, PA, USA issue in the USA, especially within un- and under-insured populations [7•]. For instance, although limited data on dermatologic health disparities exist, studies have established that minorities, individuals of lower socioeconomic status and education levels, the elderly, and uninsured patients have poorer melanoma and non-melanoma skin cancer outcomes, and atopic dermatitis is more prevalent in minorities [8]. This review highlights the ways in which teledermatology has been utilized to increase access to dermatologic care in these populations. Specifically, we discuss teledermatology's ability to reduce appointment wait times and cost-effectiveness as well as its effect on Medicaid-insured populations, rural and nonrural communities, and the elderly.

\section{Background}

Two primary models exist for teledermatology: live-interactive, which is real-time, synchronous face-to-face video visits, and store-and-forward, which is asynchronous shared digital photographs and clinical information $[9,10]$. A third hybrid model may combine real-time video with high-quality digital images [1]. In many settings, teledermatology has been shown to be a 
reliable method of diagnosing skin diseases [11-14] and results in appropriate and equivalent management plans when compared to in-person dermatology [15-17]. However, studies have found the diagnostic accuracy of teledermatology to be less than inperson visits when evaluating pigmented and cancerous lesions $[17,18 \bullet \cdot$, and it may prove difficult to replace in-person fullbody skin examinations [19]. Diagnostic concordance has generally been higher using the live-interactive model, with dramatically increased usage during the COVID-19 pandemic, compared to store-and-forward [9]. As a whole, teledermatology has widely grown in use and has been welcomed by both patients and providers $[20 \bullet, 21]$.

Teledermatology use has increased tremendously over the past several decades, with the number of US programs growing from 37 in 2011 to 102 in 2016 [22••]. According to the American Medical Association's 2016 Physician Practice Benchmark Survey, $15 \%$ of dermatologists work in settings with telemedicine [23•]. Dermatology ranks 11th among all specialties in telemedicine usage, with radiology, psychiatry, and cardiology as the top three [23•]. The greatest impact upon implementation has come from the COVID-19 pandemic in 2020 , which is likely to set precedents impacting teledermatology use long-term [24••].

\section{Shortage of Dermatologists}

A physician needs assessment in the USA calculated that is needed for every 30,000 people at least one dermatologist [25••]. Although dermatologist density increased from an estimated 1.9 per 100,000 individuals in 1970 to 3.4 in 2017 , the recommended density of 4.0 per 100,000 for adequate dermatologic care has not been met [26•]. Furthermore, the International Foundation for Dermatology has determined that 3 billion individuals residing in 345 rural communities do not have adequate dermatologic health care access [25••]. The demand for dermatologic care is increasing, and this apparent undersupply of dermatologists may be contributing to disparities in access to care. Teledermatology may serve a purpose in bridging this gap, increasing access to and efficiency of dermatology care to meet these unmet needs.

\section{Teledermatology Increases Access to Dermatologic Care}

\section{Teledermatology Reduces Patient Wait Times}

One of the largest barriers to accessing dermatologic care is difficulty obtaining appointments due to long wait times. Studies suggest that the average wait time for an appointment ranges from 18 to 33 days depending on geographic region $[27,28]$, and long wait times even occur for urgent conditions such as evolving pigmented lesions [29]. Teledermatology has the potential to circumvent these issues, by both expanding available avenues for dermatologic care and, through the use of tele-triage systems, reducing the time to in-person appointments when needed [30•, 31, 32•]. This avoidance of unnecessary in-person visits, in turn, reduces provider load and increases appointment opportunities for other patients [33].

One study found that the implementation of a teledermatology triage system significantly reduced new patient waiting times and thus led to an increase in patient access [34•]. The system also allowed for an increased total number of dermatology patient cases to be evaluated per month [34•]. In the outpatient setting, teledermatology has been associated with lower cancellations and no-show rates compared to ambulatory referrals, suggesting that patients are receiving greater levels of care with the option of teledermatology than with traditional dermatology visits alone [35••]. Teledermatology also allows referrals to occur quickly, often within hours or days, as opposed to being delayed for weeks or months after initial presentation [36•]. By increasing the number of cases that can be seen by a single dermatologist and decreasing the number of clinic visits that require in-person evaluation, teledermatology implementation intrinsically increases efficiency of care. This enhanced care delivery positively impacts patient compliance with routine skin checks and treatment regimens, allowing for early recognition and removal of suspicious lesions $[35 \bullet \bullet, 36 \bullet]$. Thus, it is unsurprising that teledermatology has been shown to decrease morbidity and mortality associated with certain skin conditions and helps to reduce negative outcomes $[35 \bullet \bullet, 37,38]$. In sum, in addition to improving efficiency, teledermatology also enhances the quality of dermatologic care in outpatient settings.

Although fewer studies have examined teledermatology's impact in hospitals, the expansion of access and efficiency does appear to improve access to care in inpatient and urgent care settings as well. Indeed, teledermatology reduces the time required for attending dermatologists to complete inpatient consultations and decreases response time of dermatologists to the primary team's initial consultation request [39]. These findings suggest that teledermatology could increase access to inpatient dermatology in settings where dermatologists have been less active in supporting their local hospitals; previously, long commuting times and the need to do lower return on investment work at offhour times, such as nights and weekends, may discourage dermatologists from offering their time and unique clinic expertise [39]. Overall, a large body of evidence has demonstrated that teledermatology increases access to care by decreasing wait times in both outpatient and inpatient settings.

\section{Cost-effectiveness of Teledermatology}

Beyond improving access given time constraints, teledermatology increases access to care without increasing 
cost [40॰]. Certainly, telemedicine typically avoids the overhead costs in the health care system that are associated with conventional in-person care, but also we must consider societal costs [20 ${ }^{\bullet}$. By decreasing the need for clinic-based visits, teledermatology can decrease patients' personal costs associated with the need to travel to clinics and missing work. A recent study demonstrated that store-and-forward teledermatology allows for shorter travel times, decreased time away from work, and faster delivery of care [33], highlighting its cost-saving benefits for patients. Although live-interactive consultations are more expensive to implement than store-and-forward teledermatology due to costs associated with video conference equipment [41], teledermatology, as a whole, has proven a cost-effective way for primary care physicians (PCPs) to refer patients to a dermatologist [42].

In addition to increasing access to specialty care, teledermatology provides educational opportunities for PCPs, which further contributes to decreasing health care system costs. One study found that PCPs who regularly participated in teledermatology referrals consequently learned how to manage common skin conditions themselves and in turn required fewer referrals [42]. As PCPs learn to independently manage common dermatologic complaints, patients can then receive treatment at earlier and more treatable stages [41]. Thus, teledermatology implementation may effectively reduce overall health care costs to society [42]. Given these combined effects of reduction of both personal and health care system costs, teledermatology not only has proven to be a clinically and diagnostically sound alternative model of care but it also appears to increase health system efficiency and cost-effectiveness, with the downstream effect of improving access.

\section{Teledermatology Decreases Health Care Disparities}

\section{Teledermatology and Medicaid}

By increasing the care opportunities, teledermatology inherently can mitigate some disparities in access, which disproportionately affect the publicly insured who often experience lower insurance acceptance rates and longer appointment wait times [43]. For example, few private dermatologists currently accept Medicaid [20•], attributed to the limited financial reimbursements. With the resulting limited availability of dermatologists and longer wait times, these Medicaid patients find it more difficult to schedule appointments and are more likely to no show, compounding the difficulty of receiving care [30 $0^{\bullet}$. Thus intuitively, telemedicine could prove useful in counteracting this access disparity. Indeed, studies have shown that teledermatology's implementation in Medicaidinsured patient populations provides significant benefits.
One large study of claims data from a California Medicaidmanaged care plan found that primary care practices engaging in teledermatology had a $64 \%$ increase in patients visiting a dermatologist compared to the $21 \%$ in other practices [44]. As a result of this study's teledermatology implementation, dermatology visits more than doubled [44], confirming that teledermatology can significantly increase access to dermatologic care among Medicaid-insured patients. The same study also found that, compared to in-person visits, teledermatology generally served healthier, younger patients with targeted and less severe skin conditions, whereas in-person dermatology visits were more neoplasm-focused [44]. Certainly, if teledermatology can effectively manage minor conditions, that can expand availability and reallocate in-person appointment slots for patients with greater need for them. At the very least, this work suggests that even if teledermatology does not necessarily avoid non-essential in-person visits, it expands access, with teledermatology Medicaid patients found to represent different demographics [44].

In addition to increasing access to care among publicly insured populations, teledermatology has been shown to improve continuity of care. A retrospective cohort study of dermatology patients in Connecticut demonstrated that teledermatology visits reduced the no-show rate among Medicaid-insured patients from 84 to $24 \%$ by providing timely face-to-face follow-up after initial virtual consult [30॰].

These results illustrate that teledermatology not only can expand access to care among Medicaid-insured patients, but it can also increase patient follow-up.

\section{Teledermatology in Non-rural Communities}

Although urban areas generally have greater dermatologist density [45••], these non-rural populations studies have disparities that teledermatology may address as well. For example, studies have shown the benefits of teledermatology in the coordination of care between PCPs and dermatologists in resource-poor, non-rural communities. One retrospective analysis of store-and-forward teledermatology consults investigated the impact of teledermatology on outpatient diagnosis, management, and access to care in 11 underserved clinics in Philadelphia [6]. PCP and dermatologist diagnoses and management plans were concordant for $22 \%$ and $23 \%$ of conditions, respectively, and the median time to consult was only 14 hours [6]. Thus, this work highlighted teledermatology's role in facilitating timely and efficient care for urban populations. Notably, at least $61 \%$ of consults in this study would not have otherwise received dermatology input, and $77 \%$ of consults were managed with teledermatology alone [6].

Teledermatology has not only increased the number of individuals receiving care in urban settings but, according to another study of store-and-forward teledermatology, has also significantly shortened wait time and reduced patient and 
provider cost in non-rural primary care practices [46]. Altogether, these studies suggest that teledermatology can efficiently provide care to outpatient populations in urban primary care settings and can expand access to care in regions even with higher dermatologist density.

\section{Teledermatology and Rural Communities}

In contrast to urban communities, rural areas typically have few dermatologists per capita [45••]. Patients from rural areas may travel up to 200 miles for a visit [47]. Further compounding the disparity in access, patients from rural areas are more likely to be older and impoverished [47]. Teledermatology has demonstrated clear potential in serving rural communities and decreasing the need for an in-person consultation. One study of pediatric store-and-forward teledermatology consultations at rural California primary care facilities found that of the 429 patients, only $1.4 \%$ required subsequent teledermatology appointments and $6.0 \%$ inperson visits [48]. Highlighting teledermatology's ability to reduce unnecessary long travel times, these results demonstrate tangible benefits among many rural US populations who otherwise have limited access to dermatologists. As a specific example, the American Indian population has historically had barriers accessing care including dermatology; on average, Indian health service centers are 68 miles from dermatology clinics and $22 \%$ of these centers do not accept Medicaid or patients without insurance [49••]. Studies have shown that this is just one, among many, of the rural populations in the USA that stands to benefit from teledermatology [49••].

Many studies have investigated teledermatology's effects on various remote populations globally. For instance, in highly rural areas without electronic medical record systems, innovative clinicians have leveraged social-networking platforms to deliver teledermatology. A study of largely inaccessible Mexican villages evaluated the use of Facebook to connect rural PCPs with a distant hospital dermatology department [50]. Photographs, relevant history, and clinical information were uploaded to Facebook and only physicians participating in the teledermatology consult from a reference hospital could view this information [50]. Approximately $75 \%$ of the 44 enrolled patients showed clinical improvement based on the treatment recommendations [50]. Teledermatology also spared these patients the long, expensive travel journeys required of them to receive care in urban centers [50]. This study demonstrated that even pre-existing networks and connections can be harnessed to create teledermatology outreach and increase access to care among rural populations.

Teledermatology has also been implemented in triage settings such as emergency departments to increase access to remote populations. In rural Australia, a pilot study at an emergency department assessed for dermatologic complaints with clinical photographs and medical histories sent both to a remote teledermatologist and face-to-face dermatology follow-up [51]. The agreement was $71.2 \%$ between teledermatology diagnosis and emergency department diagnosis, but was higher $(98 \%)$ with the in-person dermatologist [51]. Importantly, $56(93 \%)$ received a dermatology opinion within 2 hours, suggesting that teledermatology can be utilized to provide timely and medically accurate care for urgent cases in rural regions [51].

Studies have also proven the general utility of teledermatology for expanding care in the developing world, both in supporting resource-limited settings remotely and expanding the reach of the limited providers and programs in these areas. For example, one study examined a smartphone-based teledermatology program for rural communities in Kisoro, Uganda, and Lake Atitlan, Guatemala, in which local student proxies referred cases back to US dermatologists [52]. Initial diagnoses changed completely in 56\% of the 93 total cases and management changes were recommended in $89 \%$. The estimated total cost of supplies and technology per consult was low at $42.01 \mathrm{USD}$, and given the upfront costs, the cost per consult decreased with each additional case [52]. Overall, the study showed that this model of teledermatology is feasible for the delivery of care in the developing world at a relatively low cost [52]. Similarly, the African Teledermatology Project is an ongoing initiative that has implemented store-and-forward consultations in several African countries [53,54]. This program also allows for biopsy specimens to be collected and sent to the USA for processing in cases where the clinical diagnosis is difficult [53]. A study of 55 biopsies obtained through the African Teledermatology Project showed a clinicopathological concordance between submitting clinician and biopsy result in $32(58 \%)$ cases [53].

Although the implementation of teledermatology is more challenging in rural areas, many strategies have improved workflow and increased access to care: using free and easily accessible communication methods such as social networking sites, incorporating teledermatology into triage centers, and outsourcing consults to established dermatology programs in other countries.

\section{Teledermatology and the Elderly}

Teledermatology benefits resource-limited and rural areas by adding value to populations with limited in-person access. The subset of elderly patients, in particular, may be at greater risk for poor access, with traveling over even short distances posing considerable practical and economic difficulties [55]. One study of 130 geriatric dermatology patients in Sienna, Italy, investigated the efficacy of store-and-forward teledermatology compared to in-person consultations, revealing an $87 \%$ diagnostic agreement rate. These findings suggest 
that teledermatology can improve care in elderly patients who may otherwise lack easy and feasible ways to see a dermatologist [55]. Another study in Sao Paulo, Brazil, evaluated the proportion of skin lesions in patients 60 years or older that could be managed by PCP-initiated teledermatology [56*0]. This retrospective cohort study included 6633 people and found that teledermatology was adequate to avoid in-person visits in two-thirds of cases [56••].

Overall, research suggests that teledermatology may be an excellent tool in improving access to care for older populations [56••]. Indeed, for elderly patients, this method improves access to in-person dermatologic care and optimizes the use of in-person dermatology appointments for more severe, surgical, or complex diseases [56••].

\section{Teledermatology and Education}

In addition to increasing access to care for myriad underserved and marginalized communities, namely publicly insured, urban, and rural populations, and elderly patients, teledermatology can facilitate the education of students, trainees, PCPs, and dermatologists. Provider-to-provider teledermatology creates an interface for the exchange of knowledge between dermatologists and between dermatologists and other providers. Although learning may occur regardless of didactic intention, many teledermatology programs established in developing countries have included a formal educational component $[57,58]$. One study introduced a pilot curriculum in which international virtual grand rounds were conducted using teledermatology cases from Kabul, Afghanistan [59•]. This study's participants learned more about diagnosis and treatment of skin disease in international settings as well as socioeconomical and ethical issues in skin health [59•]. The use of teledermatology in Uganda and Guatemala highlighted that sustainability of teledermatology programs may depend on the education of local health care workers in order to serve as in-country dermatology consultants [52]. In that study, medical students served as proxies in this study and relayed information between local dermatologists and those in the USA. In doing so, they served as educators to local providers by conveying pertinent clinical information, diagnoses, and treatment guidelines to local providers, while learning basic principles of dermatology themselves [52].

Teledermatology can and should be simultaneously utilized in residency training programs to increase practicebased learning opportunities, while also contributing to the goal of increasing access to care. One prospective study examined diagnostic and management concordance between residents and attending dermatologists [60]. Diagnoses and management plans between resident and attending dermatologists were concordant for $53 \%$ and $65 \%$ of dermatologic conditions, respectively, highlighting a directly supervised means for residents to learn from attendings [60].

Overall, these programs have established the significant potential for education and enhanced training in dermatological diagnoses and management at all levels with the use of teledermatology.

\section{Could Teledermatology Worsen Existing Health Care Disparities?}

Although teledermatology may mitigate health care disparities, any potentially harmful effects of teledermatology must also be considered. The advancement of teledermatology that has occurred gradually over the last few decades and more exponentially over 2020 during the COVID-19 pandemic should intuitively expand access to care, given teledermatology's convenience, cost-effectiveness, and triage capabilities [61•]. However, vulnerable patients, including those of lower socioeconomic standing, older individuals, and non-English-speaking patients may experience increased barriers to accessing health care via telemedicine, particularly video visits $[62 \bullet, 63 \bullet \cdot$. The equipment needed for video visits includes smartphones, tablets, or computers, and a reliable internet connection, which can be expensive and require technological literacy. Even if an individual has access to this equipment, a reliable internet connection is not a given and has been shown to correlate with socioeconomic status. In New York City, 50\% of low-income houses lack internet access, and $26 \%$ of all Americans with an annual income of under $\$ 30,000$ rely solely on mobile internet access [64•, $65 \cdot$. Beyond digital access, telehealth services must meet quality standards, and inconsistent quality may increasingly burden vulnerable populations [63・•]. Studies have also shown that Medicaid patients are more likely to be referred for teledermatology visits and may be more vulnerable to possible diagnostic inadequacies [30•]. Thus, even as great enthusiasm continues for the potential expansion of access and reduction of disparities with teledermatology, we must remain vigilant in observing and correcting for any unintentional negative consequences.

\section{Conclusion}

Teledermatology increases access to dermatologic care, due to its cost- and time-effectiveness that benefits both providers and patients. Thus, it may prove essential for providing expanded access to many different underserved and marginalized populations in the USA and globally. Given the nation's undersupply of dermatologists, teledermatology can potentially serve as a way for dermatologists to see more patients efficiently and increase opportunities for patients to seek 
high-quality dermatology care. Teledermatology has not only enhanced patient compliance and improved individualized care but has also increased access to care among rural and urban communities both domestically and internationally. Medicaid populations in particular have benefited, but other underserved communities that stand to benefit come from rural and urban communities, and elderly populations. The implementation of teledermatology has shortened appointment wait times, increased follow-up care and treatment compliance, minimized the distance needed to be travelled for care, reduced personal and health care systemic costs, and increased dermatologic knowledge among dermatology personnel. However, teledermatology's reliance on reliable internet connections and devices could potentially worsen disparities if access to these new avenues of care is unequal.

In conclusion, teledermatology increases access to care and serves to reduce disparities among many populations given its convenience, efficiency, and cost-effectiveness. Further studies should continue investigating how to streamline expansion and implementation of teledermatology in equitable ways among all communities.

\section{Declarations}

Conflict of interest Dr. Lipoff has served as chair of the American Academy of Dermatology's Teledermatology Task Force, as a paid consultant on telemedicine for Havas Life Medicom, and as telemedicine advisor for AcneAway, a direct-to-consumer teledermatology start-up.

Human and Animal Rights and Informed Consent This article does not contain any studies with human or animal subjects performed by any of the authors.

\section{References}

Papers of particular interest, published recently, have been highlighted as:

- Of importance

•. Of major importance

1. Strehle EM, Shabde N. One hundred years of telemedicine: does this new technology have a place in paediatrics? Arch Dis Child. 2006;91(12):956-9. https://doi.org/10.1136/adc.2006.099622.

2. Kanthraj GR, Srinivas CR. Store and forward teledermatology. Indian J Dermatol Venereol Leprol. 2007;73(1):5-12. https://doi. org/10.4103/0378-6323.30643.

3. Eminović N, de Keizer NF, Bindels PJ, Hasman A. Maturity of teledermatology evaluation research: a systematic literature review. Br J Dermatol. 2007;156(3):412-9. https://doi.org/10.1111/j.13652133.2006.07627.x.

4. McKoy K, Antoniotti NM, Armstrong A, Bashshur R, Bernard J, Bernstein D, et al. Practice guidelines for teledermatology. Telemed J E Health. 2016;22(12):981-90. https://doi.org/10.1089/tmj.2016. 0137.
5. Warshaw EM, Gravely AA, Nelson DB. Reliability of store and forward teledermatology for skin neoplasms. J Am Acad Dermatol. 2015;72(3):426-35. https://doi.org/10.1016/j.jaad.2014.11.001.

6. Nelson CA, Takeshita J, Wanat KA, Bream KD, Holmes JH, Koenig HC, et al. Impact of store-and-forward (SAF) teledermatology on outpatient dermatologic care: a prospective study in an underserved urban primary care setting. J Am Acad Dermatol. 2016;74(3):484-90.e1. https://doi.org/10.1016/j.jaad. 2015.09.058.

7. Costello CM, Cumsky HJL, Maly CJ, Harvey JA, Buras MR, Pallagi PJ, et al. Improving access to care through the establishment of a local, teledermatology network. Telemed J E Health. 2020;26(7):935-40. https://doi.org/10.1089/tmj.2019.0051 Successful implementation of mobile SAF teledermatology to community health clinic outside current EHR.

8. Buster KJ, Stevens EI, Elmets CA. Dermatologic health disparities. Dermatol Clin. 2012;30(1):53-9, viii. https://doi.org/10.1016/j.det. 2011.08.002.

9. Warshaw EM, Hillman YJ, Greer NL, Hagel EM, MacDonald R, Rutks IR, et al. Teledermatology for diagnosis and management of skin conditions: a systematic review. J Am Acad Dermatol. 2011;64(4):759-72. https://doi.org/10.1016/j.jaad.2010.08.026.

10. Pak H, Triplett CA, Lindquist JH, Grambow SC, Whited JD. Storeand-forward teledermatology results in similar clinical outcomes to conventional clinic-based care. J Telemed Telecare. 2007;13(1): 26-30. https://doi.org/10.1258/135763307779701185.

11. High WA, Houston MS, Calobrisi SD, Drage LA, McEvoy MT. Assessment of the accuracy of low-cost store-and-forward teledermatology consultation. J Am Acad Dermatol. 2000;42(5 Pt 1):776-83. https://doi.org/10.1067/mjd.2000.104519.

12. Whited JD, Hall RP, Simel DL, Foy ME, Stechuchak KM, Drugge RJ, et al. Reliability and accuracy of dermatologists' clinic-based and digital image consultations. J Am Acad Dermatol. 1999;41(5 Pt 1):693-702. https://doi.org/10.1016/s0190-9622(99)70003-4.

13. Krupinski EA, LeSueur B, Ellsworth L, Levine N, Hansen R, Silvis $\mathrm{N}$, et al. Diagnostic accuracy and image quality using a digital camera for teledermatology. Telemed J. 1999;5(3):257-63. https://doi.org/10.1089/107830299312005.

14. Edison KE, Ward DS, Dyer JA, Lane W, Chance L, Hicks LL. Diagnosis, diagnostic confidence, and management concordance in live-interactive and store-and-forward teledermatology compared to in-person examination. Telemed J E Health. 2008;14(9): 889-95. https://doi.org/10.1089/tmj.2008.0001.

15. Warshaw EM, Lederle FA, Grill JP, Gravely AA, Bangerter AK, Fortier LA, et al. Accuracy of teledermatology for nonpigmented neoplasms. J Am Acad Dermatol. 2009;60(4):579-88. https://doi. org/10.1016/j.jaad.2008.11.892.

16. Loane MA, Corbett R, Bloomer SE, Eedy DJ, Gore HE, Mathews $\mathrm{C}$, et al. Diagnostic accuracy and clinical management by realtime teledermatology. Results from the Northern Ireland arms of the UK Multicentre Teledermatology Trial. J Telemed Telecare. 1998;4(2): 95-100. https://doi.org/10.1258/1357633981932028.

17. Warshaw EM, Lederle FA, Grill JP, Gravely AA, Bangerter AK, Fortier LA, et al. Accuracy of teledermatology for pigmented neoplasms. J Am Acad Dermatol. 2009;61(5):753-65. https://doi.org/ 10.1016/j.jaad.2009.04.032.

18.• Chuchu N, Dinnes J, Takwoingi Y, Matin RN, Bayliss SE, Davenport C, et al. Teledermatology for diagnosing skin cancer in adults. Cochrane Database Syst Rev. 2018;12(12):Cd013193. https://doi.org/10.1002/14651858.Cd013193 Metaanalysis evaluating teledermatology's diagnostic accuracy for malignant lesions.

19. March J, Hand M, Grossman D. Practical application of new technologies for melanoma diagnosis: Part I. Noninvasive approaches. J Am Acad Dermatol. 2015;72(6):929-41; quiz 41-2. https://doi.org/ 10.1016/j.jaad.2015.02.1138. 
20. Campagna M, Naka F, Teledermatology LJ. an updated overview of clinical applications and reimbursement policies. Int J Womens Dermatol. 2017;3(3):176-9. https://doi.org/10.1016/j.ijwd.2017. 04.002 Review highlighting reimbursement policies, costeffectiveness, and reliablility of teledermatology.

21. Weinstock MA, Nguyen FQ, Risica PM. Patient and referring provider satisfaction with teledermatology. J Am Acad Dermatol. 2002;47(1):68-72. https://doi.org/10.1067/mjd.2002.119666.

22.• Yim KM, Florek AG, Oh DH, McKoy K, Armstrong AW. Teledermatology in the United States: an update in a dynamic era. Telemed J E Health. 2018;24(9):691-7. https://doi.org/10. $1089 / \mathrm{tmj} .2017 .0253$ Identified an increase in the number and consultation volume of teldermatology programs from 20142017.

23. Kane CK, Gillis K. The use of telemedicine by physicians: still the exception rather than the rule. Health Aff (Millwood). 2018;37(12) 1923-30. https://doi.org/10.1377/hlthaff.2018.05077 This study shows a financial burden may exist when implementing telemedicine.

24.• Kennedy J, Arey S, Hopkins Z, Tejasvi T, Farah R, Secrest AM, et al. Dermatologist perceptions of teledermatology implementation and future use after COVID-19: demographics, barriers, and insights. JAMA Dermatol. In press.

25.• Coustasse A, Sarkar R, Abodunde B, Metzger BJ, Slater CM. Use of teledermatology to improve dermatological access in rural areas. Telemed J E Health. 2019;25(11):1022-32. https://doi.org/10. 1089/tmj.2018.0130 Review shows beneficial impact of teledermatology in increasing access to rural areas.

26. Glazer AM, Rigel DS. Analysis of trends in geographic distribution of US dermatology workforce density. JAMA Dermatol. 2017;153(5):472-3. https://doi.org/10.1001/jamadermatol.2016. 6032. This study examines the gerographic distribution of dermatologists in the USA.

27. Weingold DH, Lack MD, Yanowitz KL. The relative ease of obtaining a dermatologic appointment in Boston: how methods drive results. J Am Acad Dermatol. 2009;60(6):944-8. https://doi. org/10.1016/j.jaad.2009.01.022.

28. Uhlenhake E, Brodell R, Mostow E. The dermatology work force: a focus on urban versus rural wait times. J Am Acad Dermatol. 2009;61(1):17-22. https://doi.org/10.1016/j.jaad.2008.09.008.

29. Tsang MW, Resneck JS Jr. Even patients with changing moles face long dermatology appointment wait-times: a study of simulated patient calls to dermatologists. J Am Acad Dermatol. 2006;55(1): 54-8. https://doi.org/10.1016/j.jaad.2006.04.001.

30. Naka F, Lu J, Porto A, Villagra J, Wu ZH, Anderson D. Impact of dermatology eConsults on access to care and skin cancer screening in underserved populations: A model for teledermatology services in community health centers. J Am Acad Dermatol. 2018;78(2): 293-302. https://doi.org/10.1016/j.jaad.2017.09.017 A teledermatology consultant pogram with dermatoscopy increased access, reduced wait times, and providede access to skin cancer screening for underserved populations.

31. Bezalel S, Fabri P, Park HS. Implementation of store-and-forward teledermatology and its associated effect on patient access in a veterans affairs dermatology clinic. JAMA Dermatol. 2015;151(5):556-7. https://doi.org/10.1001/jamadermatol.2014. 5272.

32. Carter ZA, Goldman S, Anderson K, Li X, Hynan LS, Chong BF, et al. Creation of an internal teledermatology store-and-forward system in an existing electronic health record: a pilot study in a safety-net public health and hospital system. JAMA Dermatol. 2017;153(7):644-50. https://doi.org/10.1001/jamadermatol.2017. 0204 Epic based SAF teledermatology program improved access to dermatologic care by PCP referral.

33. Landow SM, Mateus A, Korgavkar K, Nightingale D, Weinstock MA. Teledermatology: key factors associated with reducing face- to-face dermatology visits. J Am Acad Dermatol. 2014;71(3):570 6. https://doi.org/10.1016/j.jaad.2014.02.021.

34. Zakaria A, Maurer T, Su G, Amerson E. Impact of teledermatology on the accessibility and efficiency of dermatology care in an urban safety-net hospital: a pre-post analysis. J Am Acad Dermatol. 2019;81(6):1446-52. https://doi.org/10.1016/j.jaad.2019.08.016 Teledermatology service improved access and efficency in capitated health care setting.

35.• Wang RF, Trinidad J, Lawrence J, Pootrakul L, Forrest LA, Goist $\mathrm{K}$. et al, Improved patient access and outcomes with the integration of an eConsult program (teledermatology) within a large academic medical center. J Am Acad Dermatol. 2019. https://doi.org/10. 1016/j.jaad.2019.10.053 eConsult program reveals more nonwhite patients, medicaid enrollees, and less no-shows.

36. Glazer AM, Rigel DS, Winkelmann RR, Farberg AS. Clinical diagnosis of skin cancer: enhancing inspection and early recognition. Dermatol Clin. 2017;35(4):409-16. https://doi.org/10.1016/j.det. 2017.06.001 Review emphasizing the need for early diagnosis of skin cancer and methods to do so.

37. Ludwick DA, Lortie C, Doucette J, Rao J, Samoil-Schelstraete C. Evaluation of a telehealth clinic as a means to facilitate dermatologic consultation: pilot project to assess the efficiency and experience of teledermatology used in a primary care network. J Cutan Med Surg. 2010;14(1):7-12. https://doi.org/10.2310/7750.2010.09012.

38. Whited JD, Hall RP, Foy ME, Marbrey LE, Grambow SC, Dudley TK, et al. Teledermatology's impact on time to intervention among referrals to a dermatology consult service. Telemed J E Health. 2002;8(3):313-21. https://doi.org/10.1089/15305620260353207.

39. Sharma P, Kovarik CL, Lipoff JB. Teledermatology as a means to improve access to inpatient dermatology care. J Telemed Telecare. 2016;22(5):304-10. https://doi.org/10.1177/1357633x15603298.

40. Jariwala NN, Snider CK, Mehta SJ, Armstrong JK, SmithMcLallen A, Takeshita J, et al. Prospective implementation of a consultative store-and-forward teledermatology model at a single urban academic health system with real cost data subanalysis. Telemed J E Health. 2020. https://doi.org/10.1089/tmj.2020.0248 In this study in an urban academic environment, teledermatology increased access to care without evidence of increased cost or utilization.

41. Loane MA, Bloomer SE, Corbett R, Eedy DJ, Hicks N, Lotery HE, et al. A comparison of real-time and store-and-forward teledermatology: a cost-benefit study. Br J Dermatol. 2000;143(6):1241-7. https://doi.org/10.1046/j.1365-2133.2000. 03895.x.

42. Armstrong AW, Kwong MW, Chase EP, Ledo L, Nesbitt TS, Shewry SL. Teledermatology operational considerations, challenges, and benefits: the referring providers' perspective. Telemed J E Health. 2012;18(8):580-4. https://doi.org/10.1089/tmj.2011. 0241.

43. Alghothani L, Jacks SK, Vander Horst A, Zirwas MJ. Disparities in access to dermatologic care according to insurance type. Arch Dermatol. 2012;148(8):956-7. https://doi.org/10.1001/ archdermatol.2012.804.

44. Uscher-Pines L, Malsberger R, Burgette L, Mulcahy A, Mehrotra A. Effect of teledermatology on access to dermatology care among medicaid enrollees. JAMA Dermatol. 2016;152(8):905-12. https:// doi.org/10.1001/jamadermatol.2016.0938.

45.• Feng H, Berk-Krauss J, Feng PW, Stein JA. Comparison of dermatologist density between urban and rural counties in the United States. JAMA Dermatol. 2018;154(11):1265-71. https://doi.org/ 10.1001/jamadermatol.2018.3022 Study highlights disparities in dermatology workforce between urban and rural counties.

46. McKoy KC, DiGregorio S, Stira L. Asynchronous teledermatology in an urban primary care practice. Telemed J E Health. 2004;10(Suppl 2):S-70-80. 
47. Stitzenberg KB, Thomas NE, Dalton K, Brier SE, Ollila DW, Berwick M, et al. Distance to diagnosing provider as a measure of access for patients with melanoma. Arch Dermatol. 2007;143(8):991-8. https://doi.org/10.1001/archderm.143.8.991.

48. Chen TS, Goldyne ME, Mathes EF, Frieden IJ, Gilliam AE. Pediatric teledermatology: observations based on 429 consults. J Am Acad Dermatol. 2010;62(1):61-6. https://doi.org/10.1016/j. jaad.2009.05.039.

49.• Morenz AM, Wescott S, Mostaghimi A, Sequist TD, Tobey M. Evaluation of barriers to telehealth programs and dermatological care for American Indian individuals in rural communities. JAMA Dermatol. 2019;155(8):899-905. https://doi.org/10.1001/ jamadermatol.2019.0872 Survey finds significant geographic and insurance coverage barriers to dermatology care in rural communities.

50. Garcia-Romero MT, Prado F, Dominguez-Cherit J, HojyoTomomka MT, Arenas R. Teledermatology via a social networking web site: a pilot study between a general hospital and a rural clinic. Telemed J E Health. 2011;17(8):652-5. https://doi.org/10.1089/ tmj.2011.0038.

51. Muir J, Xu C, Paul S, Staib A, McNeill I, Singh P, et al. Incorporating teledermatology into emergency medicine. Emerg Med Australas. 2011;23(5):562-8. https://doi.org/10.1111/j.17426723.2011.01443.x.

52. Greisman L, Nguyen TM, Mann RE, Baganizi M, Jacobson M, Paccione GA, et al. Feasibility and cost of a medical student proxy-based mobile teledermatology consult service with Kisoro, Uganda, and Lake Atitlán, Guatemala. Int J Dermatol. 2015;54(6): 685-92. https://doi.org/10.1111/ijd.12708.

53. Tsang MW, Kovarik CL. The role of dermatopathology in conjunction with teledermatology in resource-limited settings: lessons from the African Teledermatology Project. Int J Dermatol. 2011;50(2): 150-6. https://doi.org/10.1111/j.1365-4632.2010.04790.x.

54. Lipoff JB, Cobos G, Kaddu S, Kovarik CL. The Africa Teledermatology Project: a retrospective case review of 1229 consultations from sub-Saharan Africa. J Am Acad Dermatol. 2015;72(6):1084-5. https://doi.org/10.1016/j.jaad.2015.02.1119.

55. Rubegni P, Nami N, Cevenini G, Poggiali S, Hofmann-Wellenhof $\mathrm{R}$, Massone $\mathrm{C}$, et al. Geriatric teledermatology: store-and-forward vs. face-to-face examination. J Eur Acad Dermatol Venereol. 2011;25(11):1334-9. https://doi.org/10.1111/j.1468-3083.2011. 03986.x.

56.• M GB, Santos A, Cordioli E. Benefits of teledermatology for geriatric patients: population-based cross-sectional study. J Med Internet Res. 2020;22(4):e16700. https://doi.org/10.2196/16700 Teledermatology implementation helped to address minor complexity cases in elderly.

57. Kaddu S, Soyer HP, Gabler G, Kovarik C. The Africa Teledermatology Project: preliminary experience with a sub-
Saharan teledermatology and e-learning program. J Am Acad Dermatol. 2009;61(1):155-7. https://doi.org/10.1016/j.jaad.2008. 12.007 .

58. Desai B, McKoy K, Kovarik C. Overview of international teledermatology. Pan Afr Med J. 2010;6:3.

59. Y Yeung H, Sargen MR, Luk KM, Berry EG, Gurnee EA, Heuring E, et al. Teledermatology and teledermatopathology as educational tools for international dermatology: a virtual grand rounds pilot curriculum. Int J Dermatol. 2018;57(11):1358-62. https://doi.org/ 10.1111/ijd.14014 Virtual grand rounds provides dermatology training at an international level.

60. Nelson CA, Wanat KA, Roth RR, James WD, Kovarik CL, Takeshita J. Teledermatology as pedagogy: diagnostic and management concordance between resident and attending dermatologists. J Am Acad Dermatol. 2015;72(3):555-7. https://doi.org/10. 1016/j.jaad.2014.11.011.

61. Lee JJ, English JC 3rd. Teledermatology: a review and update. Am J Clin Dermatol. 2018;19(2):253-60. https://doi.org/10.1007/ s40257-017-0317-6 Teledermatology review examines history, evidence, and adminstrative aspects of use.

62. Eberly LA, Khatana SAM, Nathan AS, Snider C, Julien HM, Deleener ME, et al. Telemedicine outpatient cardiovascular care during the COVID-19 pandemic: bridging or opening the digital divide? Circulation. 2020. https://doi.org/10.1161/circulationaha. 120.048185 Study examining the effects of telemedicine use in cardiology during COVID-19.

63.• Bakhtiar M, Elbuluk N, Lipoff JB. The digital divide: how COVID19's telemedicine expansion could exacerbate disparities. J Am Acad Dermatol. 2020;83(5):e345-e6. https://doi.org/10.1016/j. jaad.2020.07.043 Editorial on how the COVID-19 pandemic may increase disparities due to technology access.

64. Anderson M, Kumar M. Digital divide persists even as lowerincome Americans make gains in tech adoption. Pew Research Center. 2019. https://www.pewresearch.org/fact-tank/2019/05/07/ digital-divide-persists-even-as-lower-income-americans-makegains-in-tech-adoption. Accessed 28 May 2020. Study highlights the lack of technology use in certain populations despite overall increases.

65. New York City Mayor's Office. De Blasio Administration Releases Internet Master Plan For City's Broadband Future. New York City Press Release. 2020. https://www1.nyc.gov/office-of-the-mayor/ news/010-20/de-blasio-administration-releases-internet-masterplan-city-s-broadband-future. Accessed 28 May 2020. NYC mayor plans for increased internet access.

Publisher's Note Springer Nature remains neutral with regard to jurisdictional claims in published maps and institutional affiliations. 